\title{
Inhibition of protein tyrosine phosphatase $1 B$ and regulation of insulin signalling markers by caffeoyl derivatives of chicory (Cichorium intybus) salad leaves
}

\author{
V. S. Muthusamy ${ }^{1}$, C. Saravanababu ${ }^{2}$, M. Ramanathan ${ }^{3}$, R. Bharathi Raja ${ }^{1}$, \\ S. Sudhagar ${ }^{1}$, S. Anand ${ }^{1}$ and B. S. Lakshmi ${ }^{1 *}$ \\ ${ }^{1}$ Centre for Biotechnology, Tissue Culture and Drug Discovery Laboratory, Anna University, Chennai 600 025, Tamil Nadu, India \\ ${ }^{2}$ Centre for Toxicology and Developmental Research, Sri Ramachandra Medical College and Research Institute, Chennai 600 116, \\ Tamil Nadu, India \\ ${ }^{3}$ Department of Pharmacology, PSG College of Pharmacy, Coimbatore 641 004, Tamil Nadu, India
}

(Received 28 July 2009 - Revised 23 March 2010 - Accepted 24 March 2010 - First published online 6 May 2010)

Evaluations of molecular mechanisms of dietary plants with their active molecules are essential for the complete exploration of their nutritive and therapeutic value. In the present study, we investigated the effect of chicory (Cichorium intybus) salad leaves in inhibiting protein tyrosine phosphatase 1B (PTP1B), and evaluated their role in modulating the key markers involved in insulin cell signalling and adipogenesis using 3T3-L1 adipocytes. Bioactivity-directed purification studies enlightened the additive effects of chlorogenic acid (CGA) along with other caffeic acid derivatives present in methanolic extract of $C$. intybus (CME). Incubation of CME and CGA with 3T3-L1 adipocytes significantly enhanced the 2-deoxy-D- ${ }^{3}[\mathrm{H}]$-glucose uptake and inhibited adipogenesis through altering the expressions of insulin signalling and adipogenesis markers. Extending to an in vivo model, the effect of CME was also investigated on insulin sensitivity in high-fat diet with low streptozotocin-induced diabetic rats. Supplementation of CME for 2 weeks reinstated the insulin sensitivity along with plasma metabolic profile. The present results demonstrate that the caffeoyl derivatives of chicory salad leaves show promising pharmacological effect on energy homoeostasis via PTP1B inhibition both in vitro and in vivo.

Chicory leaves: Chlorogenic acid: Protein tyrosine phosphatase 1B: Insulin resistance: Adipogenesis

Diabetes mellitus is a multifaceted disease state described by an elevated blood glucose level with reduced insulin levels, and has been observed to be frequently associated with insulin resistance, obesity, dyslipidaemia and high blood pressure ${ }^{(1)}$. Increasing pandemic of non-insulin-dependent diabetes mellitus is anticipated to rise twofold from the current estimation of 15 million by $2025^{(2)}$. Individual nutrigenomics, irregular/poor dietary choices and chronic stress are the major causes of metabolic disorders. Imbalance in the carbohydrate metabolism and the efforts of the physiological system to neutralise the changes leads to an overload of the endocrine system, leading to defects in lipid metabolism ${ }^{(3)}$. Available oral hypoglycaemic agents are devised to target various markers involved in non-insulin-dependent diabetes mellitus, but these agents have also been reported for several side effects $^{(4)}$. Currently, research into understanding the various phases of non-insulin-dependent diabetes mellitus has provided potential and specific targets for the management of non-insulin-dependent diabetes mellitus and related secondary disorders. Protein tyrosine phosphatase 1B (PTP1B) has emerged as a potential target for diabetes and obesity because of its central association in insulin signalling and adipogenesis ${ }^{(5)}$. For a normal physiological condition, a fine balance is essential between the kinases such as insulin receptor tyrosine kinase, which are involved in downstream insulin signals, and the phosphatases such as PTP1B, which are required to shut down these signals ${ }^{(6)}$. But the discovery of PTP1B inhibitors with selectivity, bioavailability and acceptable pharmacokinetic properties has proven to be extremely difficult ${ }^{(7)}$.

In the UK diet, salad foods such as Cichorium intybus are preferred in a higher ratio than vegetables such as onion, broccoli and green beans for the management of various chronic disorders $^{(8)}$. In our earlier study, we have reported the PTP1B inhibitory activity of methanolic extract of $C$. intybus (CME), and investigated its role in glucose transport and adipogenesis in 3T3-L1 adipocytes ${ }^{(9)}$. In the present paper, we extend our analysis to investigate the active molecules

Abbreviations: CGA, chlorogenic acid; CME, Cichorium intybus methanolic extract; DMEM, Dulbecco's modified Eagle's medium; G6P, glucose-6-phosphatase; GSK, glycogen synthase kinase; HFD, high-fat diet; IR, insulin receptor; IRS, IR substrate; PI3K, phosphatidylinositol 3-kinase; Akt/PKB, protein kinase B; PC, plasma cholesterol; PG, plasma glucose; PT, plasma TAG; PTP1B, protein tyrosine phosphatase 1B; STZ, streptozotocin.

* Corresponding author: B. S. Lakshmi, fax +91 44 22350299, email lakshmibs@annauniv.edu 
present in $C$. intybus responsible for PTP1B inhibition. Maintaining the bioavailability of PTP1B inhibitors has been reported to be a difficult task for the reason that PTP require an inhibitor with more electronegative pharmacophores for their deactivation ${ }^{(10)}$. Hence, we have also examined the effect of CME on plasma biochemical parameters and carbohydrate metabolism markers of high-fat diet with low streptozotocin (HFD/STZ)-induced type 2 diabetic male Wistar albino rats. The present study also discusses important insights into the additive effects of caffeoyl derivatives present in $C$. intybus on PTP1B inhibition.

\section{Materials and methods}

\section{Chemicals and reagents}

Dulbecco's modified Eagle's medium (DMEM) and serum (fetal bovine serum) were obtained from GIBCO-BRL (Gaithersburg, MD, USA). 2-Deoxy-D- ${ }^{3}[\mathrm{H}]$-glucose was obtained from Amersham Pharmacia Biotech (Little Chalfont, Bucks, UK). Insulin, 3-isobutyl-1-methylxanthine and dexamethasone were obtained from Sigma-Aldrich (St Louis, MO, USA). TRIzol reagent and murine leukemia virus reverse transcriptase, dNTP and Taq polymerase were obtained from GIBCO-BRL and New England Biolabs (Hitchin, UK), respectively. All antibodies were obtained from Calbiochem (Darmstadt, Germany). Rosiglitazone was a kind gift from Dr. Reddys Laboratories (Hyderabad, India). Recombinant human PTP1B was obtained from Calbiochem. Sephadex LH-2 was obtained from Pharmacia (Stockholm, Sweden). High-performance TLC stationary plates (Silica gel 6GF254) were obtained from Merck India (Darmstadt, Germany). Plasma glucose (PG), cholesterol (PC), TAG (PT) and insulin were measured using commercially available diagnostic kits (Accurex, Bangalore, India) in a semi-automatic biochemical analyser (Merck 200). All the chemicals and reagents used were of analytical grade. Leaves of $C$. intybus were collected from Trichy, Tamil Nadu, India. The collected material has been authenticated, and the vouchers are stored at the Tissue Culture and Drug Discovery Laboratory, Anna University, Chennai, India.

\section{Animals}

Male Wistar albino rats $(160-180 \mathrm{~g})$ were procured from the Central Animal House, PSG college of Pharmacy, Coimbatore, India, and were acclimatised to laboratory conditions for a period of 1 week. Rats were housed in polypropylene cages (five to six rats/cage) under an ambient temperature of $23 \pm 3^{\circ} \mathrm{C}$ and $40-65 \%$ relative humidity, with $12-12 \mathrm{~h}$ light and dark cycle. They were maintained on a standard pellet diet and water ad libitum before the dietary manipulation. Guidelines of Guide for the Care and Use of Laboratory Animals (Institute of Laboratory Animal Resources, National Academic Press, 1996; NIH publication \#85-23, revised 1996) were strictly followed throughout the study. Experimental protocol was approved by the Institutional Animal Ethical Committee, Anna University, Chennai, India (IAEC/CBT/ Min. dt. 06.10.08/Protocol No. 7).

\section{Cell culture of 3T3-L1 adipocytes}

3T3-L1 preadipocytes (ATCC, Manassas, VA, USA; passage number 5 or lower) were cultured in DMEM with $1 \%$ fetal bovine serum. 3T3-L1 preadipocytes grown in twenty-fourwell plates were induced using the differentiation medium (combination of 3-isobutyl-1-methylxanthine, dexamethasone and insulin in DMEM with $1 \%$ fetal bovine serum) to differentiate into adipocytes. After differentiation, the medium was replaced with insulin medium (DMEM with $1 \%$ fetal bovine serum containing $1 \mathrm{mg} / \mathrm{ml}$ of insulin) for $2 \mathrm{~d}$. The extent of differentiation was established by observing the multinuclei of cells.

\section{Extraction, bioactivity-guided isolation and high-performance} TLC studies

CME (5 gm) was prepared as described previously ${ }^{(9)}$, and was subjected to solvent-solvent fractionation by dissolving in $60 \%$ methanol. The soluble portion of CME in $60 \%$ methanol was partitioned with hexane, chloroform and butanol. Based on the bioactivity, a butanol-soluble fraction $(1 \mathrm{~g})$ was selected and submitted to further purification by column chromatography on Sephadex LH-2 using a chloroform-methanol gradient mobile phase system (from ratio 10:9 to 50:50), which afforded five subfractions. Subfraction 4 gave $21.2 \mathrm{mg}$ of yellow-coloured amorphous powder with significant 2-deoxy-D- ${ }^{3}[\mathrm{H}]$-glucose uptake activity. The pure molecule was subjected to NMR and mass spectroscopic studies for structure elucidation. High-performance TLC was performed according to the method elaborated earlier ${ }^{(11)}$. The stock solution of CME $(10 \mathrm{mg} / \mathrm{ml})$ and chlorogenic acid (CGA) standard $(1 \mathrm{mg} / \mathrm{ml})$ was used for TLC identification/quantification of CME. The following chromatographic conditions were applied. Stationary phase, silica gel $\mathrm{GF}_{254}$; mobile phase, ethyl acetate-acetic acid-formic acid-water (100:11:11:25, by vol.); instrument, high-performance TLC instrument (Camag, Muttenz, Switzerland; version 1.3.4); applicator, Linomat V; scanner, Camag TLC scanner III; detection scanning wavelength, $330 \mathrm{~nm}$. The limit of detection and limit of quantification were estimated using isolated pure CGA. For quantitative analysis, CME was chromatographed and scanned at $330 \mathrm{~nm}$. The amount of CGA present in CME was calculated by comparing the absorption units of standard CGA.

\section{Measurement of 2-deoxy- $\mathrm{D}^{3}[\mathrm{H}]$-glucose and adipogenesis inhibition assay}

3T3-L1 adipocytes grown in twenty-four-well plates (BD Falcon, Rock Hill, SC, USA) were subjected to glucose uptake as reported $^{(12)}$ with minor modifications. Differentiated adipocytes were subjected to serum starvation for $5 \mathrm{~h}$ followed by incubation of the plant extracts for $24 \mathrm{~h}$. After incubation, cells were stimulated with insulin $(100 \mathrm{nM})$ for $20 \mathrm{~min}$ followed by incubation for $20 \mathrm{~min}$ in Krebs Ringer phosphate solution containing $0.018 \mathrm{MBq} / \mathrm{ml}$ 2-deoxy-D- ${ }^{3}[\mathrm{H}]$-glucose. All the assays were performed in duplicates for concordance, and compared with known positive control (rosiglitazone $50 \mu \mathrm{M})$. Results were expressed as percentage change in glucose uptake with respect to the solvent control. The effect of CME and CGA on 3T3-L1 preadipocyte differentiation was 
assessed by measuring the accumulation of $\mathrm{TAG}^{(13)}$. Preadipocytes were maintained in fresh DMEM every other day for the comparison of TAG accumulation. Photomicroscopic evaluation was carried out to qualitatively measure the TAG content using a commercially available kit (AdipoRed assay Reagent; Lonza Walkersville, Inc., Walkersville, MD, USA).

\section{Protein tyrosine phosphatase $1 B$ inhibition study}

The PTP1B inhibition of CME and CGA (from $1 \mathrm{pg}$ to $10 \mathrm{mg}$ ) was carried out using $p$-nitrophenyl phosphate as the substrate in a ninety-six-well format ${ }^{(7)}$. Sodium orthovanadate $(100 \mathrm{~mm})$ was used as a positive control. The rate of PTP1B-catalysed hydrolysis of $20 \mathrm{~mm}$ - $p$-nitrophenyl phosphate was measured by following the absorbance change at $405 \mathrm{~nm}$.

\section{$R T-P C R$}

RT-PCR was performed according to the method described previously $^{(9)}$. The RNA obtained from 3T3-L1 adipocytes after experimental incubation was converted to complementary DNA by reverse transcription, and subjected to PCR with specific primers for CCAAT/enhancer-binding protein, PPAR $\gamma$, sterol-regulatory element-binding protein-1c and glyceraldehyde 3-phosphate dehydrogenase. PCR products were run on $1.5 \%$ agarose gels, stained with ethidium bromide and photographed. To determine the efficacy, insulin and rosiglitazone were used at $100 \mathrm{nM}$ and $50 \mu \mathrm{M}$ doses, respectively, for all the markers.

\section{Immunoprecipitation and immunoblotting}

Whole-cell lysate ${ }^{(14)}$ and tissue extracts ${ }^{(15)}$ were prepared as reported previously with minor modifications. Total protein $(100 \mu \mathrm{g})$ isolated from the tissues of the experimental animals was mixed with a substrate-soluble buffer, and heated at $90-100^{\circ} \mathrm{C}$ for $10 \mathrm{~min}$ followed by $1 \%$ SDS-polyacrylamide gel elution and electrophoretic transfer to a nitrocellulose membrane. For insulin receptor substrate (IRS-1), immunoprecipitation was performed before electrophoresis. For the purpose of blocking, the membrane was stored overnight in a blocking agent $\left(5 \%\right.$ skimmed milk) at $4^{\circ} \mathrm{C}$. The blot was washed with PBS Tween and then with PBS. Then, the membrane was incubated with the desired primary antibody (PTP1B, IR, IRS-1, phosphatidylinositol 3-kinase (PI3K), protein kinase B (Akt/PKB), glycogen synthase kinase (GSK)-3 $\beta$, GLUT4 and $\beta$-actin) for $2 \mathrm{~h}$. After washing, the membrane was incubated with alkaline phosphatase-conjugated secondary antibody in $1 \%$ bovine serum albumin in PBS for $1 \mathrm{~h}$ at room temperature. Then, the blot was washed with PBS thrice, each $5 \mathrm{~min}$, and the blot was developed with a chromogenic agent and photographed.

\section{Development of high-fat diet-fed and streptozotocin-treated type 2 diabetic rats}

Experimental animals were allocated to two dietary regimens, either normal pellet diet or HFD. After 3 weeks of HFD regimen, STZ ( $35 \mathrm{mg} / \mathrm{kg}$, intra peritonial) was administered to the experimental animals ${ }^{(16)}$. Blood sample was collected after $72 \mathrm{~h}$ through a sino-orbital puncture, and PG, PC and PT levels were measured. Experimental animals with $\mathrm{PG} \geq 2500 \mathrm{mg} / \mathrm{l}$ and $\mathrm{PC} \geq 2000 \mathrm{mg} / \mathrm{l}$ were used for the study. Experimental animals were divided into three groups as follows: group I - normoglycaemic (normal pellet diet) - received citrate buffer + vehicle $(0.5 \%$ carboxy methyl cellulose); group II - hyperglycaemic (HFD) - received STZ + vehicle $(0.5 \%$ carboxy methyl cellulose); group III - hyperglycaemic (HFD) received STZ + CME $(125 \mathrm{mg} / \mathrm{kg}$, per os $)$. From the dose range-finding study, $125 \mathrm{mg} / \mathrm{kg}$ body weight was found to be an optimal dose (based on the efficacy, body weight and behavioural parameters; data not shown). Treatment was scheduled for $14 \mathrm{~d}$ following induction of diabetes. PG, PC and PT were measured on days 0,7 and 14 of the experiment according to the manufacturers protocol using a semi-automatic biochemical analyser (Merck 200). Plasma insulin was measured using an ELISA reader (Accurex Diagnostic kit) following the manufacturers instruction. On day 14, following blood collection, the experimental animals were euthanised to collect the tissues, and the tissues were stored at $-80^{\circ} \mathrm{C}$ until analysis.

\section{Biochemical assay of glycogen content and enzyme activities}

Liver and skeletal muscle glycogen content was estimated using a previously elaborated method ${ }^{(17)}$. The rats were euthanised, and tissue samples were collected for the assessment of biochemical parameters. The following enzymes were evaluated as described previously glucose-6-phosphatase $(\mathrm{G} 6 \mathrm{P})^{(18)}$ and fructose-1,6-diphosphatase ${ }^{(19)}$.

\section{Statistical analysis}

The data obtained from the in vitro studies are expressed as means with their standard errors. Mean difference between the groups was analysed by one-way ANOVA followed by Tukey's multiple comparison test (using SPSS version 11.0 software; SPSS, Cary, NC, USA). Statistical analysis was performed using GraphPad Prism, 4.03 (San Diego, CA, USA). Data are expressed as means with their standard errors. Unpaired Students $t$ test was used for analysing the group mean difference in body weight and glucose, cholesterol and TAG parameters. One-way ANOVA followed by Dunnett's post hoc test was used for analysing other biochemical parameters. $P<0.05$ was fixed as the statistical significance criterion.

\section{Results \\ Bioactivity-guided isolation of active molecule and high-performance TLC quantification}

CME was subjected to solvent-solvent fractionation, and the hydroalcohol-insoluble $(0.64 \mathrm{~g})$, hexane-soluble $(0.2 \mathrm{~g})$, chloroform-soluble $(0 \cdot 19 \mathrm{~g})$, butanol-soluble ( $1 \cdot 72 \mathrm{~g})$ and hydroalcohol-soluble $(2.05 \mathrm{~g})$ fractions that were obtained were subjected to 2-deoxy-D- ${ }^{3}[\mathrm{H}]$-glucose uptake studies (Fig. 1(a)). Based on the glucose uptake activity, butanol-soluble fraction was selected for column purification, which yielded CGA as an active molecule (Fig. 1(b)). The purity of CGA isolated from CME was confirmed by comparing the UV absorption spectra. Based on the spectral characterisation studies, the pure molecule was identified to be a $\mathrm{CGA}^{(20)}$. Spectral characters of the isolated compound were found to be UV $(\mathrm{MeOH})$ 


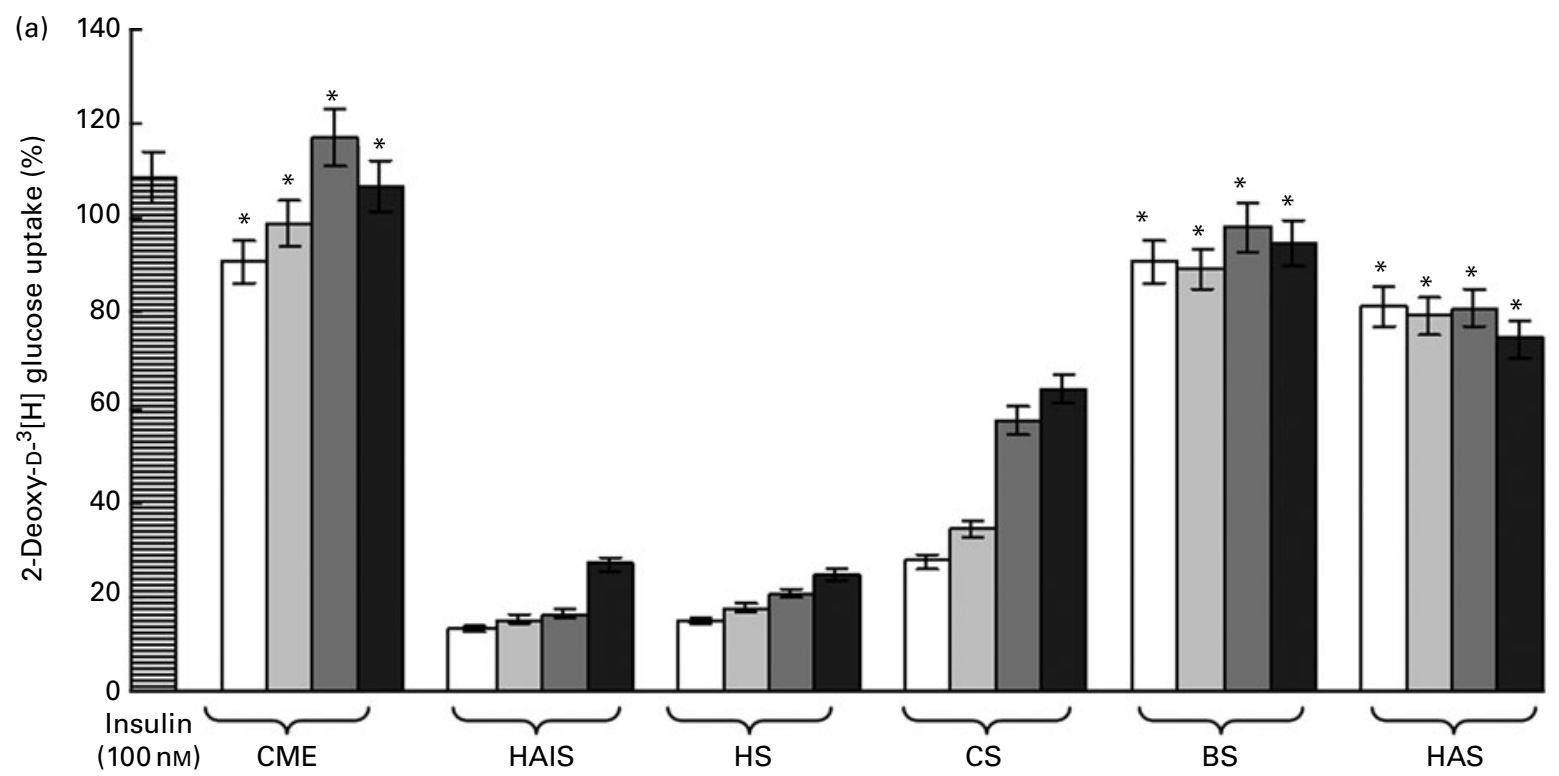<smiles>O=C(/C=C/c1ccc(O)c(O)c1)OC1CC(O)(C(=O)O)CC(O)C1O</smiles>

(c)

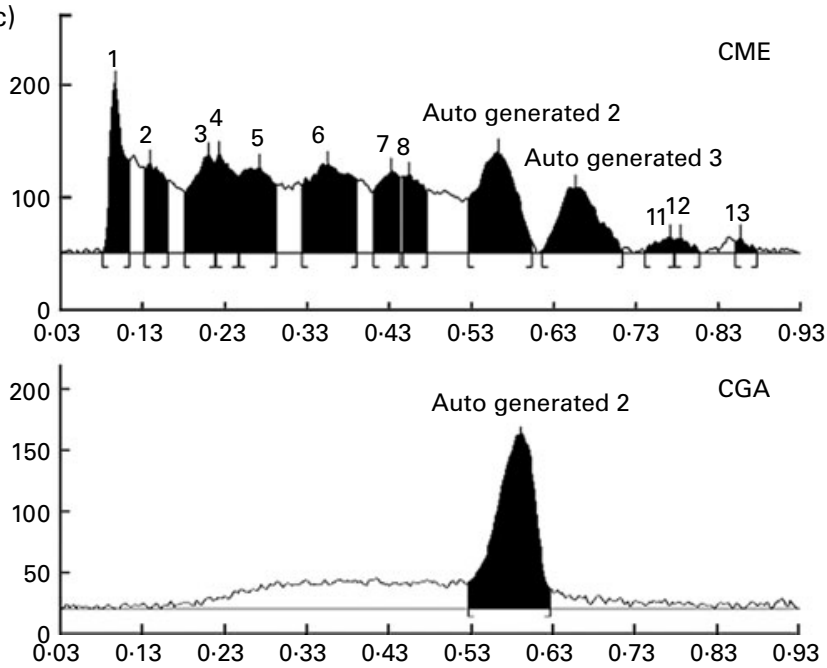

Fig. 1. (a) 2-Deoxy-D- ${ }^{3}[\mathrm{H}]$-glucose uptake activities of the fractions obtained from methanolic extract of Cichorium intybus (CME; HAIS, hydroalcohol insoluble; HS, hexane soluble; CS, chloroform soluble; BS, butanol soluble; HAS, hydroalchol soluble). Differentiated 3T3-L1 adipocytes were preincubated with described doses of fractions for $24 \mathrm{~h}$. The cells were subjected to $15 \mathrm{~min}$ insulin stimulation $(10 \mathrm{nM})$, and then $0.018 \mathrm{MBq} / \mathrm{ml}$ of 2 -deoxy-D- ${ }^{3}[\mathrm{H}]-$ glucose was added for 20 min and the uptake was measured. The results are expressed as percentage change in glucose uptake with respect to the solvent control (dimethyl sulphoxide). Data are expressed as means with their standard errors, $n 3 .{ }^{*}$ Mean values were significantly when compared with the untreated control group $(P<0.05)$. (b) Structure of chlorogenic acid (CGA) compound isolated from $C$. intybus through bioactivity-guided fractionation and (c) high-performance TLC chromatograms of CME and CGA at $330 \mathrm{~nm}$. (a) $\square, 10 \mathrm{ng} / \mathrm{ml} ; \square, 100 \mathrm{ng} / \mathrm{ml} ;(\square), 1 \mu \mathrm{g} / \mathrm{ml} ; \mathbf{\square}, 10 \mu \mathrm{g} / \mathrm{ml}$.

$\lambda_{\max } 228,257$ and $330 \mathrm{~nm},{ }^{1} \mathrm{H}$ NMR $\left(\mathrm{CD}_{3} \mathrm{OD}\right) \delta 7 \cdot 58(1 \mathrm{H}, \mathrm{d}$, H-7), 7.07 (1H, d, Hz, H-2), 6.90 (1H, dd, H-6), 6.74 (1H, d, H-5), $6 \cdot 3$ (1H, d, H-8), $5 \cdot 3\left(1 \mathrm{H}, \mathrm{m}, \mathrm{H}-3^{\prime}\right), 4 \cdot 19\left(1 \mathrm{H}, \mathrm{m}, \mathrm{H}-5^{\prime}\right)$, $3.72\left(1 \mathrm{H}, \mathrm{dd}, \mathrm{H}-4^{\prime}\right), 2 \cdot 15-2 \cdot 25\left(2 \mathrm{H}, \mathrm{m}, \mathrm{H}-6^{\prime}\right), 2 \cdot 00-2 \cdot 10(2 \mathrm{H}$, $\left.\mathrm{m}, \mathrm{H}-2^{\prime}\right) .{ }^{13} \mathrm{C}-\mathrm{NMR}\left(500 \mathrm{MHz}, \mathrm{CD}_{3} \mathrm{OD}\right) \delta: 167 \cdot 2(\mathrm{C}-9), 76 \cdot 5$ (C-8), 145.1 (C-7), $128 \cdot 2$ (C-6), $115 \cdot 1$ (C-5), $144 \cdot 8$ (C-4), $143 \cdot 4$ (C-3), $117 \cdot 8$ (C-2), $120 \cdot 0$ (C-1), $36 \cdot 8\left(\mathrm{C}-6^{\prime}\right), 69 \cdot 8$ $\left(\mathrm{C}-5^{\prime}\right), 71 \cdot 8\left(\mathrm{C}-4^{\prime}\right), 70 \cdot 2\left(\mathrm{C}-3^{\prime}\right), 35 \cdot 2\left(\mathrm{C}-2^{\prime}\right), 74 \cdot 6\left(\mathrm{C}-1^{\prime}\right), 177 \cdot 2$ (C-carbonyl). Electronspray ionisation-MS (negative ion mode) $\mathrm{m} / z, 353[\mathrm{M}-\mathrm{H}]^{-}$. The chromatographic analysis of CME and isolated CGA was carried out at $330 \mathrm{~nm}$, and they were photographed at long UV (Fig. 1(c)). In the present study, the limit of detection and limit of quantification for CGA were found to be $200 \mathrm{ng}$ and $1 \mu \mathrm{g}$, respectively. The amount of CGA present in CME was found to be $1.2 \% \mathrm{w} / \mathrm{w}$ (dry weight basis).
Effect of methanolic extract of Cichorium intybus and chlorogenic acid on 2-deoxy-D- ${ }^{3}[H]$-glucose uptake and insulin cell signalling

CME and CGA (from $1 \mathrm{pg}$ to $10 \mu \mathrm{g}$ ) were analysed for radiolabelled glucose uptake efficiency. A dose-dependent increase (Fig. 2(a)) in glucose uptake was observed with both CME and CGA. Maximum uptake was observed for CME and CGA at concentrations of 10 and $10 \mathrm{ng} / \mathrm{ml}$, respectively. The cell cytotoxicity of CGA was studied at various concentrations ranging from $1 \mathrm{pg}$ to $10 \mu \mathrm{g}$, and it was found to be non-toxic (data not shown). The time-dependent effect of CME $(100 \mathrm{ng} / \mathrm{ml})$ and CGA $(10 \mathrm{ng} / \mathrm{ml})$ on IR $\beta$, IRS-1, PI3K, Akt/PKB, GSK-3 $\beta$ and GLUT4 protein expressions was observed (Fig. 2(c)-(h)) from $24 \mathrm{~h}$ onwards and continued till $36 \mathrm{~h}$ treatment. 
No significant difference was observed in the expression of PTP1B upon treatment with CME and CGA (Fig. 2(b)).

Effect of methanolic extract of Cichorium intybus and chlorogenic acid on adipogenesis

To evaluate the adipogenesis inhibitory potential of CME and CGA, co-treatment of CME $(100 \mathrm{ng} / \mathrm{ml})$ and CGA $(10 \mathrm{ng} / \mathrm{ml})$ with the differentiation medium was performed. The changes in lipid accumulation were calculated (Fig. 3(a)) and photomicrographed at $10 \times$ magnification (Fig. 3(b)-(e)). Timecourse RT-PCR analysis revealed the suppressive effect of CME and CGA on mRNA expression of key adipogenic genes CCAAT/enhancer-binding protein, PPAR $\gamma$ and sterolregulatory element-binding protein-1c (Fig. 3(f)-(h)), and the effect was observed from $18 \mathrm{~h}$ onwards and continued till $24 \mathrm{~h}$ treatment. The levels of adipogenic genes were expressed after normalising with GAPDH (Fig. 3(i)).

\section{Protein tyrosine phosphatase $1 B$ inhibition study}

As demonstrated in Fig. 4, CME and CGA showed a dose-dependent inhibition of PTP1B enzyme activity. The $\mathrm{IC}_{50}$ of $\mathrm{CME}$ and CGA was found to be $21.2 \mathrm{ng}$ and $3 \cdot 12 \mu \mathrm{g}$, respectively.

Effect of methanolic extract of Cichorium intybus on body weight and feed intake

There was a significant decrease in body weight in the vehicletreated hyperglycaemic group than in the normoglycaemic group during the experimental period (Fig. 5(a)). Surprisingly,

(a)

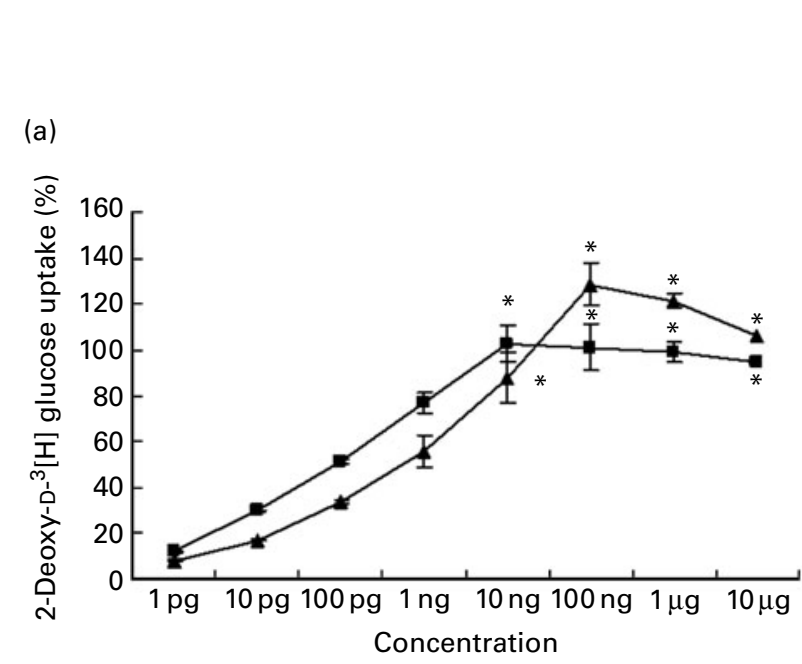

the CME-treated group also showed a significant decrease in body weight on days 7 and $14(P<0.05$ and $P<0.01$, respectively) than the vehicle-treated normoglycaemic group. A similar observation was made in the food intake behaviour of the experimental animals (Fig. 5(b)). Dietary intake of the CME-treated group was decreased significantly $(P<0.05)$ than that of the normoglycaemic group.

Effect of methanolic extract of Cichorium intybus on plasma blood glucose, plasma cholesterol, plasma TAG and plasma insulin levels

Dietary manipulation plus low dose of STZ significantly $(P<0 \cdot 01)$ elevated PG, PC, PT and plasma insulin levels in the experimental animals. The normal pellet diet-fed rats appeared normal throughout the experimental period. Table 1 shows the effect of CME on PG, PC, PT and plasma insulin levels. The biochemical levels were found to be significantly $(P<0.01)$ high in hyperglycaemic group than in the normoglycaemic group on day 0 . Oral administration of CME significantly decreased PG, cholesterol and TAG levels measured on days 7 and $14(P<0.05$ and 0.01 , respectively). But interestingly, CME-treated group showed a non-significant increase in insulin levels during the experimental period.

Effect of methanolic extract of Cichorium intybus on the expression of protein tyrosine phosphatase $1 B$ and other insulin signalling markers in the muscles of experimental animals

There was a significant increase in PTP1B expression in skeletal muscles of the hyperglycaemic rats than in those of the normoglycaemic rats, and a moderate restoration was

(b)

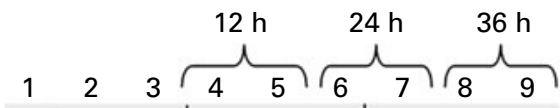

(c)
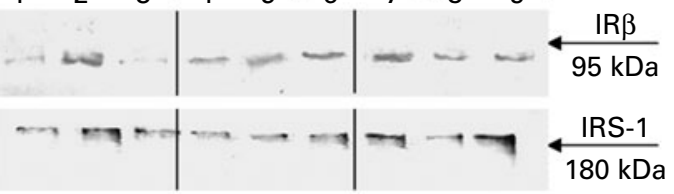

(d)

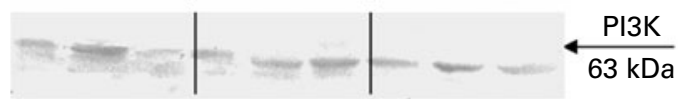

(e)

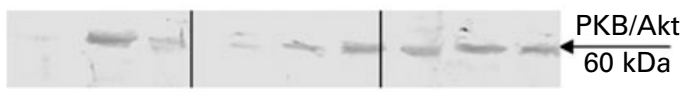

(f)

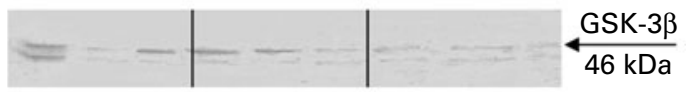

(g)

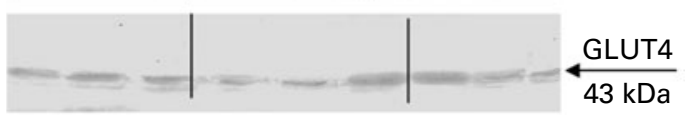

(h)

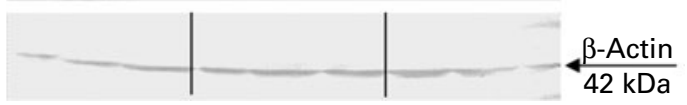

Fig. 2. (a) Dose-response analysis of methanolic extract of Cichorium intybus (CME, $-\mathbf{\Delta -}$ ) and chlorogenic acid (CGA, $-\mathbf{-}-)$ in 2-deoxy-D- $\left.{ }^{3} \mathrm{H}\right]-\mathrm{glucose}$ uptake activity. After experimental incubation of CME and CGA with differentiated 3T3-L1 adipocytes, the cells were subjected to 15 min insulin stimulation (10 nm). After buffer washing, 2-deoxy-D- ${ }^{3}[\mathrm{H}]$-glucose $(0.018 \mathrm{MBq} / \mathrm{ml})$ was added to the cells for $20 \mathrm{~min}$, and the uptake was measured. Data are expressed as means with their standard errors, $n$ 3. * Mean values were significantly different when compared with the untreated control group $(P<0.05)$. (b) $-(\mathrm{g})$ Western blot analysis of insulin signalling markers insulin receptor $\beta$ (IR $\beta$ ), insulin receptor substrate (IRS-1), phosphatidylinositol 3-kinase (PI3K), protein kinase B (PKB/Akt), glycogen synthase kinase (GSK-3 $\beta$ ) and GLUT4 using whole-cell lysate of CME- and CGA-treated 3T3-L1 adipocytes at different time points from 12 to $36 \mathrm{~h}$. (h) Protein expressions were normalised by $\beta$-actin. 

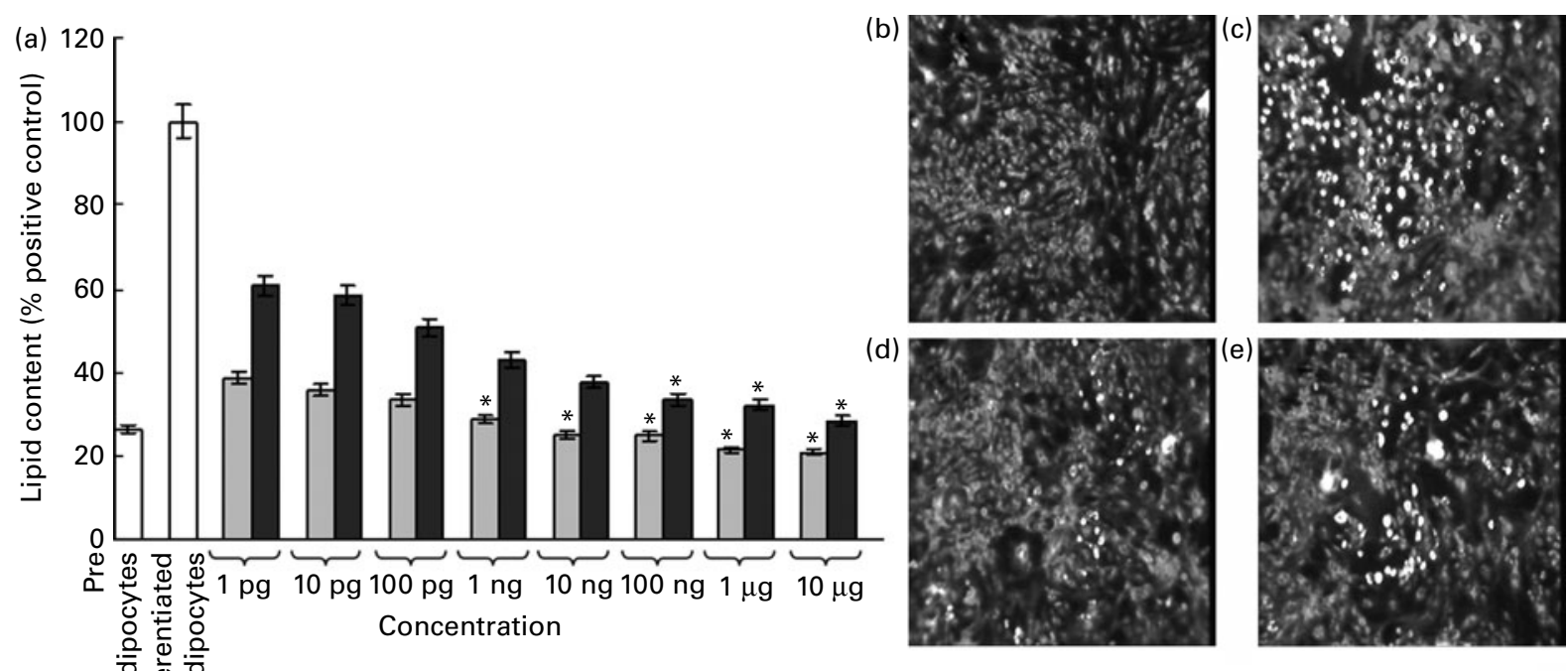

它
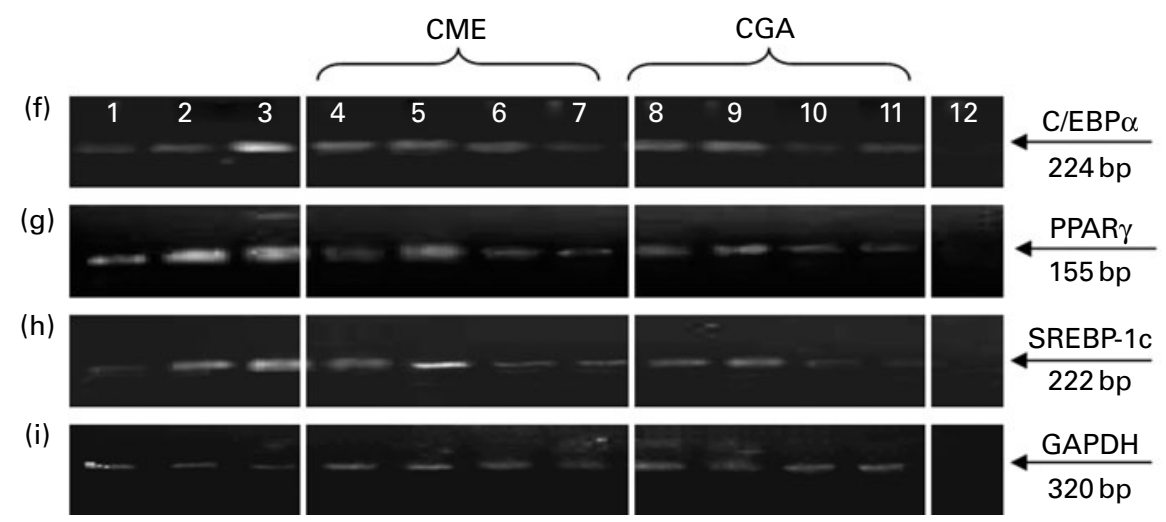

Fig. 3. Effect of methanolic extract of Cichorium intybus (CME, $\square$ ) and chlorogenic acid (CGA, $\mathbf{\square}$ ) on adipogenesis. (a) CME and CGA suppress lipid accumulation in maturing preadipocytes during incubation for a whole spectrum of differentiation periods. Preadipocytes were separately maintained using a protocol as indicated. Data that are shown reflect the means with their standard errors of triplicates of two determinations. ${ }^{\star}$ Mean values were significantly different when compared with the preadipocyte group $(P<0.05)$. Cellular TAG was stained with the AdipoRed ${ }^{\circledR}$ reagent, and the fluorescent lipid droplets (white dots) were photomicrographed at $10 \times$ magnification. (b) Control; (c) differentiation induced; (d) differentiation medium + CME (100 ng/ml); (e) differentiation medium + CGA $(100 \mathrm{ng} / \mathrm{ml})$. (f) $-(\mathrm{h})$ RT-PCR analysis of adipogenic genes CCAAT/enhancer-binding protein (C/EBP $\alpha, 224 \mathrm{bp})$, PPARg (155 bp) and sterol-regulatory element-binding protein (SREBP-1c, $222 \mathrm{bp}$ ) at different time points (624h) in CME- and CGA-treated 3T3-L1 adipocytes. (i) mRNA of glyceraldehyde 3-phosphate dehydrogenase (GAPDH) in the same sample was used as a loading reference.

recorded upon treatment with CME (Fig. 6(a)). Insulin downstream signalling markers such as IR, PI3K, PKB, GSK-3 $\beta$ and GLUT4 were also significantly reinstated in the CME-treated rats (Fig. 6(b)-(f)). The protein expressions were normalised with $\beta$-actin (Fig. $6(\mathrm{~g})$ ).

Effect of methanolic extract of Cichorium intybus on hepatic and skeletal muscle glycogen content

After $14 \mathrm{~d}$ of treatment, the experimental animals were euthanised, and hepatic and skeletal muscle glycogen content was determined. Significant decrease in hepatic $(F(3,20)=40 \cdot 35$, $P<0.01)$ and skeletal muscle $(F(3,20)=17 \cdot 83, P<0 \cdot 01)$ glycogen content was observed in the vehicle-treated hyperglycaemic rats than in the normoglycaemic rats. Administration of $\mathrm{CME}$ for $14 \mathrm{~d}$ increased glycogen content in liver $(P<0.01)$ and skeletal muscle $(P<0.05)$ when compared with the hyperglycaemic rats (Fig. 7(a) and (b)).
Effect of methanolic extract of Cichorium intybus on key enzymes involved in carbohydrate metabolism

Effect of CME on hepatic G6P and fructose-1,6-diphosphatase enzymes in the hyperglycaemic rats is shown in Fig. 8(a) and (b). Induction of type 2 diabetes significantly increased the hepatic G6P $(F(3,20)=5 \cdot 86, \quad P<0.01)$ and fructose-1,6diphosphatase $(F(3,20)=10.83, P<0.01)$ activities in rats. Administration of $\mathrm{CME}$ for $14 \mathrm{~d}$ decreased G6P and fructose-1,6-diphosphatase activities.

\section{Discussion}

PTP1B has been reported to play the role of a central switch in controlling insulin signalling and adipogenesis ${ }^{(21)}$. In our earlier study, the biological role of $C$. intybus crude extract in PTP1B inhibition was ascertained in adipocytes ${ }^{(9)}$. 3T3-L1 adipocyte cell culture model of adipogenesis has been reported to be a reliable model to study lipid metabolism 


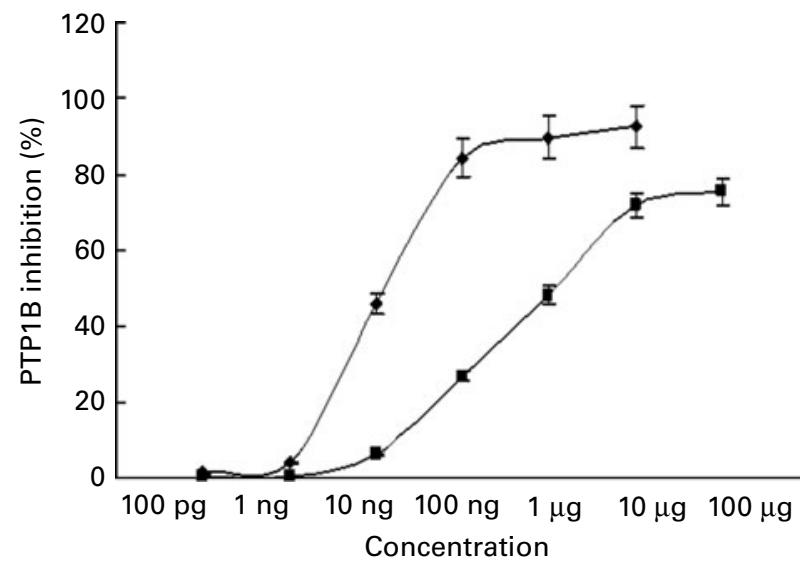

Fig. 4. Effect of methanolic extract of Cichorium intybus (CME, - -) and chlorogenic acid (CGA, - - - ) on inhibition of protein tyrosine phosphatase 1B (PTP1B) enzyme. CME and CGA showed a dose-dependent inhibition of PTP1B enzyme with the $I_{50}$ of $21.2 \mathrm{ng}(--)$ and $3.82 \mathrm{mg}(-\mathbf{-})$, respectively.

and insulin cell signalling in vitro ${ }^{(22)}$. Green \& Kehinde ${ }^{(23)}$ have reported that a major limitation of the 3T3-L1 model is the loss of the differentiation ability of the preadipocytes with variation in cell copy number when passaged extensively.

Taking this into consideration, 3T3-L1 adipocytes with low passage number $(<5)$ were used in the present study. As an extension, the present study was intended to evaluate the key molecule (s) present in $C$. intybus which could be responsible for PTP1B inhibition and in vivo efficacy. The key molecule responsible for the PTP1B inhibitory effect of CME was identified to be CGA. Interestingly, although the dose-response analysis of 2-deoxy-D- ${ }^{3}[\mathrm{H}]$-glucose uptake and insulin signalling markers revealed that CGA acts through a mechanism that is similar to that through which CME acts, the activity of CME was better than that of the active molecule (CGA) obtained through bioactivity-guided purification. Usually, therapeutic superiority of a crude extract over a single isolated molecule would be caused by additive effects of the other molecules present in the extract ${ }^{(24)}$. Li et al. ${ }^{(25)}$ have also observed a similar dominance in the anti-cancer activity of Chinese traditional herb Coptis chinensis with its active molecule berberine. One of the major problems in regularising diet is the identification of key molecules responsible for its activity, because most of the dietary plants function additively or synergistically ${ }^{(26)}$. Even in the present study, no significant improvement in the glucose uptake, adipogenesis and PTP1B inhibitory activities was observed during the enrichment of CME.

Cinnamic acid and caffeic acid, the precursors of all caffeoyl derivatives ${ }^{(27)}$, have been well studied for glucose uptake $^{(28)}$ and PTP1B inhibitory activities ${ }^{(29)}$. C. intybus contains very low quantities ${ }^{(30)}$ of cinnamic acid and caffeic acid that eliminate the possibility of their influence on the net activity of CME. Chicoric acid a dicaffeoyl ester from C. intybus is present in substantial amounts ${ }^{(27)}(4.5-6.5 \%$, $\mathrm{w} / \mathrm{w})$, but it has been reported for moderate $(<20 \%$ uptake) glucose-transporting activity ${ }^{(31)}$. Therefore, we hypothesise that CGA would be the most active molecule among all the caffeic acid derivatives present in $C$. intybus exhibiting glucose uptake activity, whereas the association of other caffeoyl derivatives such as cinnamic acid, caffeic acid and chicoric acid would be adding to the net biological activity of CME. Hence, CGA was selected as a chemical and biological marker for the standardisation of the $C$. intybus extract. Rationalising the therapeutic efficacy of a medicinal plant with its most active constituent has been considered to be a reasonable approach for investigating herbal preparations ${ }^{(32)}$. To standardise CME, a simple and robust quantitative highperformance TLC method was also developed. High-performance TLC studies revealed that CME contains $1.219 \% \mathrm{w} / \mathrm{w}$ of CGA, which is comparable with earlier reports ${ }^{(26)}$.

PTP1B has been reported to be a key modulator of downstream markers of insulin signal transduction such as IR, IRS, PI3K and $\mathrm{PKB}^{(21)}$. Insulin-stimulated GLUT4 translocation and the fate of glucose in translating into glycogen are mainly mediated through $\mathrm{PI} 3 \mathrm{~K}^{(33)}$. Significant increase in IR, IRS, PI3K and PKB expressions along with a subsequent decrease in GSK-3 $\beta$ expression evidently substantiates PTP1B inhibition-mediated glucose-transporting mechanism of CME and CGA. Rondinone et al. ${ }^{(34)}$ observed a decrease in adipogenesis with a downregulation of all adipogenic genes in animals ablated with PTP1B, and postulated the pivotal role of PTP1B in the development of obesity. The quantitative adipogenesis analysis and photomicrographs of cells incubated with CME and CGA clearly revealed the variation in lipid droplet formations. The present results on
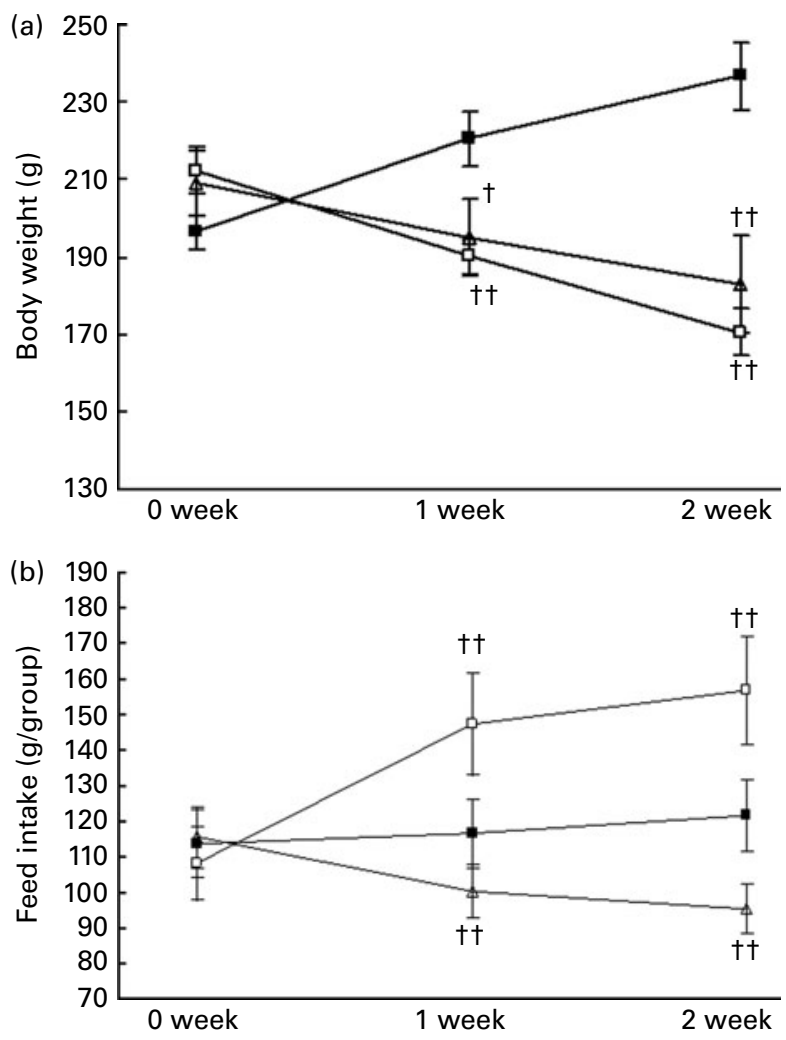

Fig. 5. Effect of methanolic extract of Cichorium intybus (CME, $125 \mathrm{mg} / \mathrm{kg}$ body weight) on (a) body weight and (b) feed intake behaviour of experimental rats. Values are expressed as means with their standard errors, $n 6$ Mean values were significantly different from those when compared with the normoglycaemic group $(-\square-)$ : $† P=0.05$, $\dagger \dagger P=0.01$. $-\square-$, Hyperglycaemic group; $\triangle$, hyperglycaemic + CME group. 
(a)

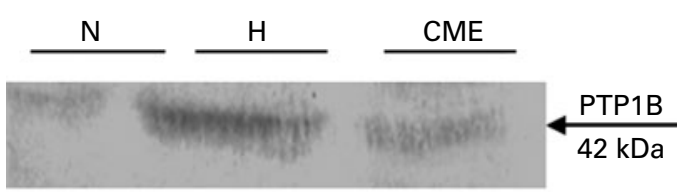

(b)

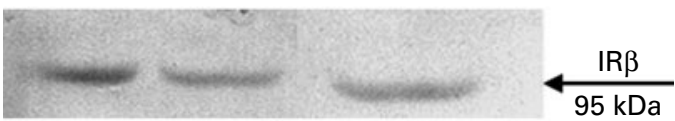

(c)

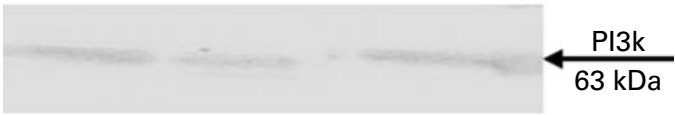

(d)

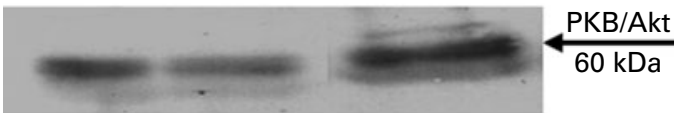

(e)

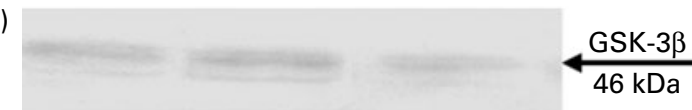

(f)

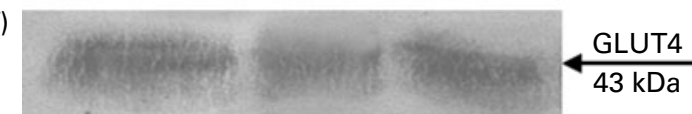

(g)

$\frac{\beta \text {-Actin }}{42 \mathrm{kDa}}$

Fig. 6. (a)-(f) Effect of methanolic extract of Cichorium intybus (CME) administration (125 mg/kg body weight) on protein tyrosine phosphatase $1 \mathrm{~B}$ (PTP1B), insulin receptor (IRß), phosphatidylinositol 3-kinase (PI3K), protein kinase $B$ (PKB/Akt), glycogen synthase kinase (GSK-3ß) and GLUT4 expressions in rat skeletal muscle, respectively. (g) Protein expressions were normalised by $\beta$-actin. $\mathrm{N}$, normoglycaemic; $\mathrm{H}$, hyperglycaemic; CME, CME treated.

CCAAT/enhancer-binding protein $\alpha$, PPAR $\gamma$ and sterol-regulatory element-binding protein-1c expressions coincide with our earlier findings, and corroborate the PTP1B inhibitory mechanism of CME and CGA.

One of the major hindrances in developing PTP1B inhibitors has been the identification of a small molecule with bioavailability. To address this, we analysed the in vivo efficacy of CME in diabetic rats. HFD in combination with a low-dose STZ (35 mg/kg body weight)-induced diabetes model has been reported to show similar metabolic features of insulin resistance ${ }^{(35)}$, and has been used for the evaluation of PTP1B inhibitors ${ }^{(36)}$. Pushparaj et al. ${ }^{(37)}$ have identified the intestinal glucose absorption-decreasing property of inulin isolated from $C$. intybus. But no reports were available regarding the evaluation of $C$. intybus in insulin-resistant animal model. Additionally, the clustal analysis of the homology of PTP1B between rats and human was observed to be more than $8 \%$ (www.ebi.ac.uk/clustal - data not shown). Hence, we adapted the HFD- and low-dose STZinduced rat model to evaluate the in vivo efficacy of CME.

In the experimental conditions, a significant decrease in body weight was observed upon treatment with CME in diabetic rats. A similar observation was reported earlier for vanadium salts, which are known inhibitors of PTP1B ${ }^{(38)}$. Additionally, a consistent decrease in food intake was also noticed in the CME-treated rats. PTP1B controls energy metabolism and appetite by modulating leptin levels both 

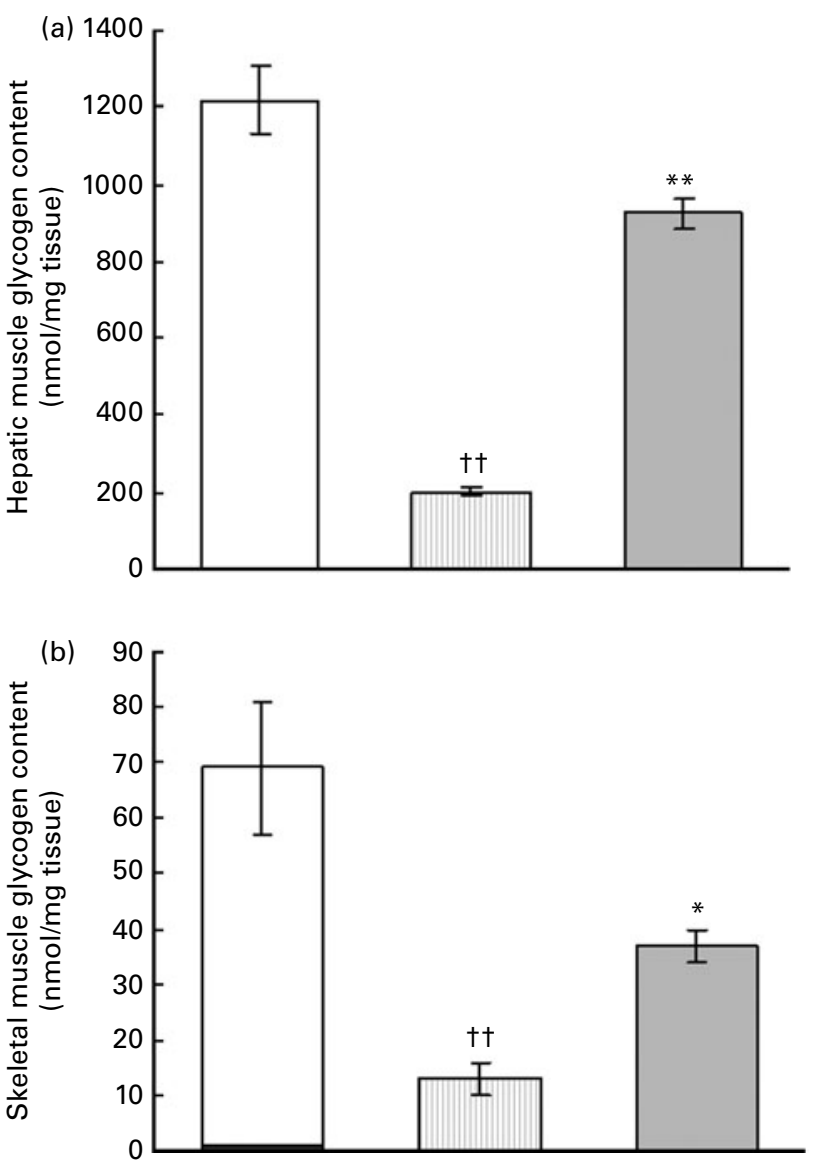

Fig. 7. Effect of methanolic extract of Cichorium intybus (CME) treatment on (a) hepatic and (b) skeletal muscle glycogen content. Values are expressed as means with their standard errors, $n$. Mean values were significantly different from those when compared with the hyperglycaemic group (四): ${ }^{\star} P=0.05{ }^{* \star} P=0.01$. Mean values were significantly different from those when compared with the normoglycaemic group $(\square)$ : $\dagger \dagger P=0.05$. $\square$, Hyperglycaemic + CME group.

peripherally and centrally. PTP1B inhibitors with a capability to cross the blood-brain barrier are expected to control body weight and appetite ${ }^{(39)}$. The ability of CGA to cross blood-brain barrier has been well documented ${ }^{(40)}$. Additionally, the $\log P$ values of other molecules such as cinnamic acid and caffeic acid fall at an optimal $\log P$ range $(<1.5)$ to cross the blood-brain barrier ${ }^{(41)}$. Hence, the observation can be correlated with PTP1B inhibitory efficacy of CGA and other caffeoyl derivatives present in CME. Administration of CME produced potent anti-diabetic activity in the HFD/ STZ rats as evidenced by decreased PG, PC and PT levels, which can be corroborated to its efficacy in PTP1B inhibition. A non-significant increase in insulin levels with $\mathrm{CME}$ treatment can be rationalised by the hepatic G6P inhibitionmediated insulinotropic effect of caffeoyl derivatives ${ }^{(31)}$.

Furthermore, to confirm the PTP1B inhibition-mediated mechanism of $\mathrm{CME}$ in influencing insulin signalling markers, the protein expressions in the skeletal muscle of HFD/STZ-treated type 2 diabetic rats were analysed. The function of PTP1B is tissue specific, and its role in skeletal muscles and adipocytes has been well elucidated ${ }^{(42)}$. Hence, skeletal muscle was used in the present study. Up-regulation of IR, PI3K, PKB and GLUT4 and suppression of GSK-3 $\beta$ with CME treatment that were observed through immunoblotting demonstrate the PTP1B-mediated insulinomimetic action of CME.

Inhibition of negative regulators of insulin signalling cascade such as PTP1B has been reported to direct the energy utilisation towards glycogen storage ${ }^{(5)}$. Lochhead et al. ${ }^{(43)}$ postulated the link between activation of PI3K and $\mathrm{PKB}$ and a decrease in the transcriptions of gluconeogenic enzymes such as G6P and F16BP. In the present study, increase in skeletal muscle glycogen content by CME clearly shows that it acts through the modulation of PTP1B, PI3K and GSK-3 $\beta$. Since liver glycogen levels have also been regulated by hepatic G6P enzyme, the current observation can also be reasoned to be due to the G6P inhibitory effects of caffeoyl derivatives (CGA and cichoric acid) present in $C$. intybus ${ }^{(31)}$. Biostability is one of the major limitations of the PTP1B inhibitor drug development. Since cinnamic acid and caffeic acid are the major metabolites of $\mathrm{CGA}^{(44)}$, we hypothesise that the metabolic process may not alter the activity of CGA.

Currently, nutrigenomics and dietary intervention studies with new targets involved in energy homoeostasis are gaining attention for the management of metabolic disorders. The clinical significance of PTP1B has been well documented

(a)
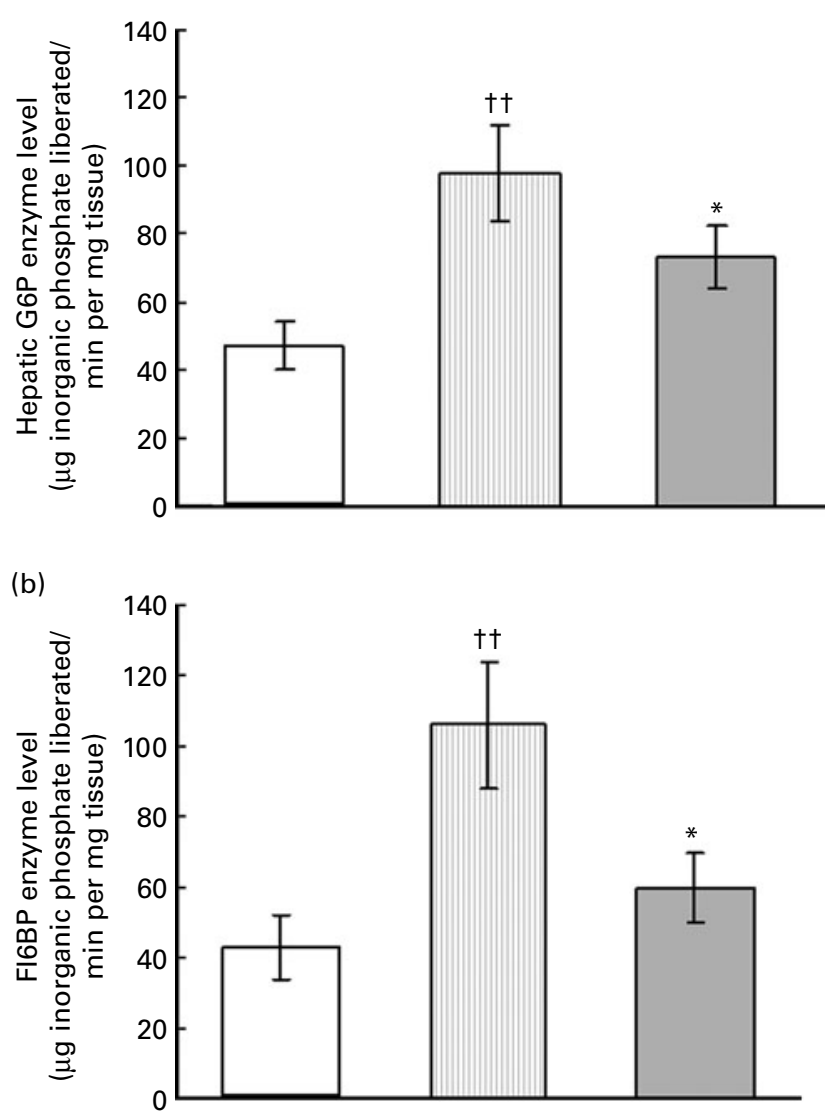

Fig. 8. Effect of methanolic extract of Cichorium intybus (CME) on (a) hepatic glucose-6-phosphatase (G6P) and (b) F16BP enzyme levels in diabetic rats. Value are expressed as means with their standard errors, $n 6$. Mean values were significantly different from those when compared with the hyperglycaemic group $(\mathbb{W l})$ : ${ }^{*} P=0.01$. Mean values were significantly different from those when compared with the normoglycaemic group $(\square)$ : $\dagger+P=0.05$. $\square$, Hyperglycaemic + CME group. 
by evidencing its association in inhibiting insulin signal transduction. The present study has revealed the ability of commonly eaten salad leaves $C$. intybus in normalising glucose metabolism without altering lipid metabolism through the PTP1B inhibitory mechanism.

\section{Acknowledgements}

B. S. L. and V. S. M. conceptualised and designed the study, and wrote the manuscript. C. S. and M. R. helped in the in vivo experiments. R. B. R. and S. S. helped in the bioactivity-guided fractionation studies. V. S. M., C. S. and S. A. collated all statistical information. All authors read and approved the findings of the study. There is no conflict of interest. V. S. M. is a CSIR - Research Associate and is highly grateful to the Council of Scientific and Industrial Research, Government of India, for the financial assistance.

\section{References}

1. Saltiel AR (2001) New perspectives into the molecular pathogenesis and treatment of type 2 diabetes. Cell 104, 517-529.

2. Zimmet P, Alberti KG \& Shaw J (2001) Global and societal implications of the diabetes epidemic. Nature 414, 782-787.

3. Shulman GI (2000) Cellular mechanisms of insulin resistance. $J$ Clin Invest 106, 171-176.

4. Izumi T \& Tomohiro K (2004) Future therapy of diabetes mellitus. Biomed Pharmacother 58, 578-581.

5. Byon JC, Kusari AB \& Kusari J (1998) Protein-tyrosine phosphatase-1B acts as a negative regulator of insulin signal transduction. Mol Cell Biochem 182, 101-108.

6. Gum RJ, Gaede LL, Koterski SL, et al. (2003) Reduction of protein tyrosine phosphatase $1 \mathrm{~B}$ increases insulin-dependent signaling in $o b / o b$ mice. Diabetes 52, 21-28.

7. Wiesmann C, Barr KJ, Kung J, et al. (2004) Allosteric inhibition of protein tyrosine phosphatase 1B. Nat Struct Mol Biol 11, 730-737.

8. Hertog MGL, Hollman PCH, Katan MB, et al. (1993) Intake of potentially anticarcinogenic flavonoids and their intake in adults in The Netherlands. Nutr Cancer 20, 21-29.

9. Muthusamy VS, Anand S, Sangeetha KN, et al. (2008) Tannins present in Cichorium intybus enhance glucose uptake and inhibit adipogenesis in 3T3-L1 adipocytes through PTP1B inhibition. Chem Biol Interact 174, 69-78.

10. Zhang ZY, Wang Y, Wu L, et al. (1994) The Cys(X)5Arg catalytic motif in phosphoester hydrolysis. Biochemistry 33, $15266-15270$.

11. Saravanababu C, Sunil AG, Vasanthi HR, et al. (2007) Development and validation of an HPTLC method for simultaneous estimation of excitatory neurotransmitters in rat brain. $J$ Liq Chromatogr Relat Technol 30, 2891-2902.

12. Liu X, Kim JK, Li Y, et al. (2005) Tannic acid stimulates glucose transport and inhibits adipocyte differentiation in 3T3-L1 cells. J Nutr 135, 165-171.

13. Ambati S, Kim HK, Yang JY, et al. (2007) Effects of leptin on apoptosis and adipogenesis in 3T3-L1 adipocytes. Biochem Pharmacol 73, 378-384.

14. Klip A, Guma A, Ramlal T, et al. (1992) Stimulation of hexose transport by metformin in L6 muscle cells in culture. Endocrinology 130, 2535-2544.

15. Thirone AC, Carvalheira JB, Hirata AE, et al. (2004) Regulation of $\mathrm{Cbl}$-associated protein/Cbl pathway in muscle and adipose tissues of two animal models of insulin resistance. Endocrinology 145, 281-293.
16. Srinivasan K, Viswanad B, Asrat L, et al. (2005) Combination of high-fat diet-fed and low-dose streptozotocin-treated rata model for type 2 diabetes and pharmacological screening. Pharmacol Res 52, 313-320.

17. Nicholas V (1956) The determination of glycogen in liver and muscle by use of anthrone reagent. Indian J Biol Chem 220, 583-587.

18. Baginski ES, Foa PP \& Zak B (1967) Determination of rat liver microsomal glucose-6-phosphatase activitystudy of citrate and G-6-P inhibition. Anal Biochem 21, 201-207.

19. Gancedo JM \& Gancedo C (1971) Fructose-1,6-diphosphatase, phospho fructokinase and glucose-6-phosphate dehydrogenase from fermenting and non fermenting yeasts. Arch Mikrobiol 76, 132-138.

20. Pauli GF, Kuczkowiak U \& Nahrstedt A (1999) Solvent effects in the structure dereplication of caffeoyl quinic acids. Magn Reson Chem 37, 827-836.

21. Ukkola O \& Santaniemi M (2002) Protein tyrosine phosphatase 1B: a new target for the treatment of obesity and associated co-morbidities. J Intern Med 251, 467-475.

22. Rajala MW \& Scherer PE (2003) Minireview: the adipocyte at the crossroads of energy homeostasis, inflammation, and atherosclerosis. Endocrinology 144, 3765-3773.

23. Green H \& Kehinde O (1975) An established preadipose cell line and its differentiation in culture. II. Factors affecting the adipose conversion. Cell 5, 19-27.

24. Wagner H (2005) Natural products chemistry and phytomedicine in the 21st century: new developments and challenges. Pure Appl Chem 77, 1-6.

25. Li X, Motwani M, Tong W, et al. (2000) Huanglian, a Chinese herbal extract, inhibits cell growth by suppressing the expression of cyclin $\mathrm{B} 1$ and inhibiting $\mathrm{CDC} 2$ kinase activity in human cancer cells. Mol Pharmacol 58, 1287-1293.

26. Davis CD (2007) Nutritional interactions: credentialing of molecular targets for cancer prevention. Exp Biol Med 232, $176-183$.

27. Innocenti M, Gallori S, Giaccherini C, et al. (2005) Evaluation of the phenolic content in the aerial parts of different varieties of Cichorium intybus L. J Agric Food Chem 53, 6497-6502.

28. Jung UJ, Lee MK, Park YB, et al. (2006) Antihyperglycemic and antioxidant properties of caffeic acid in $d b / d b$ mice. $J$ Pharmacol Exp Ther 318, 476-483.

29. Lakshmi BS, Sujatha S, Anand S, et al. (2009) Cinnamic acid, from the bark of Cinnamomum cassia, regulates glucose transport via activation of GLUT4 on L6 myotubes in a phosphatidylinositol 3-kinase-independent manner. J Diabetes 1, 99-106.

30. Molgaard P \& Ravn H (1988) Evolutionary aspects of caffeoyl ester distribution in dicotyledons. Phytochem 27, 2411-2421.

31. Tousch D, Lajoix AD, Hosy E, et al. (2008) Chicoric acid, a new compound able to enhance insulin release and glucose uptake. Biochem Biophys Res Commun 377, 131-135.

32. Winslow LC \& Kroll DJ (1998) Herbs as medicines. Arch Intern Med 158, 2192-2199.

33. Kenner KA, Anyanwu E, Olefsky JM, et al. (1996) Proteintyrosine phosphatase 1B is a negative regulator of insulin- and insulin-like growth factor-I-stimulated signaling. $\mathrm{J}$ Biol Chem 271, 19810-19816.

34. Rondinone CM, Trevillyan JM, Clampit J, et al. (2002) Protein tyrosine phosphatase $1 \mathrm{~B}$ reduction regulates adiposity and expression of genes involved in lipogenesis. Diabetes 51, 2405-2411.

35. Proietto J, Filippis A, Nakhla C, et al. (1999) Nutrient-induced insulin resistance. Mol Cell Endocrinol 151, 143-149.

36. Wu Y, Ou-Yang JP, Wu K, et al. (2005) Hypoglycemic effect of Astragalus polysaccharide and its effect on PTP1B. Acta Pharmacol Sin 26, 345-352. 
37. Pushparaj PN, Low HK, Manikandan J, et al. (2007) Anti-diabetic effects of Cichorium intybus in streptozotocininduced diabetic rats. J Ethnopharmacol 111, 430-434.

38. Meyerovitch J, Shechter Y \& Amir S (1989) Vanadate stimulates in vivo glucose uptake in brain and arrests food intake and body weight gain in rats. Physiol Behav 45, 1113-1116.

39. Morrison CD (2008) Leptin resistance and the response to positive energy balance. Physiol Behav 94, 660-663.

40. Lapchak PA (2007) The phenylpropanoid micronutrient chlorogenic acid improves clinical rating scores in rabbits following multiple infarct ischemic strokessynergism with tissue plasminogen activator. Exp Neurol 205, 407-413.

41. Summerfield SG, Read K, Begley DJ, et al. (2007) Central nervous system drug disposition: the relationship between in situ brain permeability and brain free fraction. J Pharmacol Exp Ther 322, 205-213.

42. Goldstein BJ (2002) Protein-tyrosine phosphatases: emerging targets for therapeutic intervention in type 2 diabetes and related states of insulin resistance. J Clin Endocrinol Metab 87, 2474-2480.

43. Lochhead PA, Coghlan M, Rice SQ, et al. (2001) Inhibition of GSK-3 selectively reduces glucose-6-phosphatase and phosphatase and phosphoenolypyruvate carboxykinase gene expression. Diabetes 50, 937-946.

44. Moridani MY, Scobie H, Jamshidzadeh A, et al. (2001) Caffeic acid, chlorogenic acid, and dihydrocaffeic acid metabolismglutathione conjugate formation. Drug Metab Dispos 29, 1432-1439. 